\title{
Research on the Parametric Data Acquisition Technology for Rural Real Estate Fieldwork
}

\author{
Xiaohui Huang ${ }^{1, a}$, Xiaoguo Zhang ${ }^{2, b, *}$ and Baohong Feng ${ }^{2, c}$
}

${ }^{1}$ School of Geomatics Science and Technology, Nanjing Tech University, Nanjing 211816, China.

${ }^{2}$ School of Instrument Science and Engineering, Southeast University, Nanjing 210096, China.

ah09x25h@126.com, ${ }^{*}$, bxy519@sina.com, cfbhhll@163.com

\begin{abstract}
Rural houses are frequently changed and the data update takes much time and effort. Concerning the lack of software for real estate surveying and mapping fieldwork, and the difference of interaction between mobile devices and ordinary computer, the parametric data acquisition technology for rural real estate fieldwork is presented in this paper. Firstly, the technology flow of parametric automatic mapping based on screen hand-drawn sketches is designed. Then, the key algorithms of automatic recognition of hand-drawn sketch structures, automatic construction of dimension chains, and automatic parametric drawing are overcome. Finally, the Android platform software with the above functions is developed. And the experiment is designed to verify the functions and performance of the system. The results show that the technology can realize the realtime mapping, and can improve the mapping fieldwork efficiency.
\end{abstract}

Keywords: house map, data acquisition of fieldwork, software design, parametric plotting, housing database.

\section{Introduction}

Real estate registration is a matter of national economy and people's livelihood [1]. One important achievement of real estate survey is the house map, which has large quantities [2]. Traditional house mapping survey consists of two modes of operation: Artificial mapping mode and computer-aided mapping mode. Both are based on field measurement data. The former records the dimensions on a sketch, which is more intuitive. The disadvantage is that it requires manual recording of measurement data and manual calculation of building construction area, which is easy to make mistakes and it is also difficult to meet the needs of the engineering unit. On the premise of the field measurement data, the latter uses AutoCAD, CASS and real estate mapping software to complete mapping work, and does not improve the efficiency of the field measurement work.

At present, there are many mature real estate mapping software in the domestic market. These software can perform area calculation and map drawing according to the requirements of the real estate measurement specification. However, the area measurement method determines that the calculation locations need to be separated from the field site, and the separation of the office and field work leads to the low efficiency and the error prone.

In view of the previous situation, the development of the real estate house map software based on the synchronized mapping of sketches is put forward in this paper. First, the requirement analysis of the fieldwork mapping software is carried out, and the interactive fieldwork data collection technology process of the mobile terminal is designed. Then, key algorithms such as automatic sketch drawing recognition, the automatic construction of the dimension chain and the parametric selfconstruction are studied and realized. Finally, the Android platform software with the above functions is developed. While data was collected in the field, parameterization was plotted under the sketch synchronization diagram. By uploading the digital results of the fieldwork in real time, the efficiency of the real estate measurement in China is greatly improved. 


\section{System Work Flow and Software Architecture Design}

\subsection{Software Requirement Analysis.}

The field mapping work needs to observe the actual situation of the house to draw the sketches, record the measured data on the sketches, process the image, and finally calculate the housing area.

The problems discovered during the analysis of field operations include:

(1) The speed of sketching directly affects the speed of measurement, and the mapping habits of surveyors influence the readability of sketches.

(2) In order to keep the accuracy of the dimension records, it needs to be time-consuming to modify when mistakes are found.

(3) The surveyors are familiar with the house conditions. It is more appropriate for the same surveying and mapping technician to perform from the collection of field data to the office mapping and area calculation. If the time interval is too long, it is easy to be forgot.

In order to make it convenient for surveyors to draw house graphics and record house information in real time and accurately. it is necessary to develop mapping software on mobile devices. This software is oriented to the real estate house map, and is mainly used for the follows:

(1) Quickly draw sketches in field measurements.

(2) Quickly collect and record house data.

(3) Free to modify the size and dimension driven drawing.

(4) Automatically redraw the house graphics in the change measurement.

(5) Automatically generate the house map and area statistics.

The hand-held device has the characteristics of mouse free and touch screen, and can provide an interaction mode different from ordinary computers [3]. The interactive process based on the handheld device is shown in Figure 1.

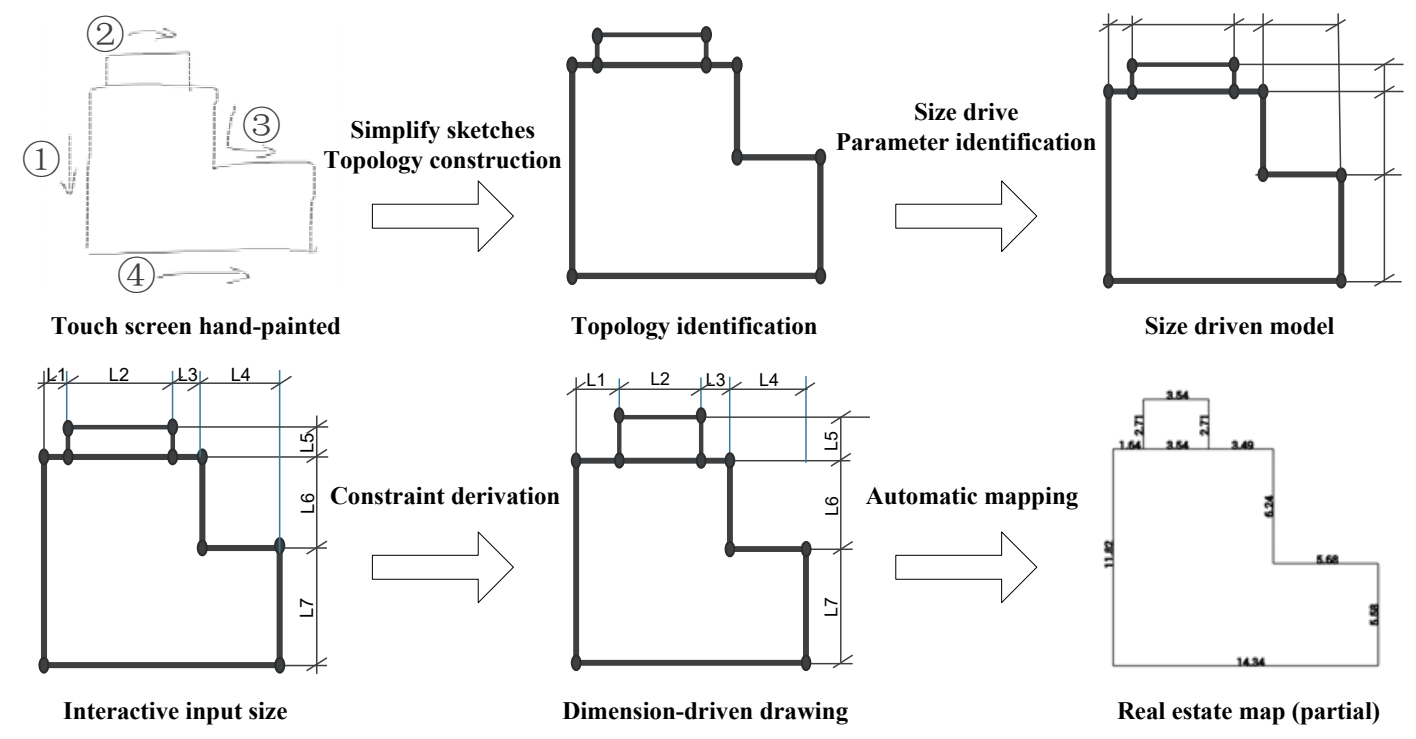

Fig. 1 Mapping Interactive Process of Hand-Held Device

It has the following main functions:

(1) Screen sketch. According to the geometry of house, the surveyor draws the sketches on the intelligent terminals.

(2) Automatic mapping of sketches. The software organizes sketches into regular and realistic house graphs and identifies its topology.

(3) Interactive marking. Use the range finder to measure the size, and pick up any house contour line to input field measurement data.

(4) Dimension driven drawing. Derive the dimensional constraints according to the dimensional parameters and modify graphs. 
(5) Automatic reports generation. When the complete data is available, the program synchronizes the area to the database, displays the graph and data in the report, and generates the house map.

Object oriented method is used for requirement analysis. The corresponding Use case diagram for the function of the field mapping software can be represented as Figure 2. There are three roles: measuring staff, mobile terminals, databases, these roles involve many different Use cases.

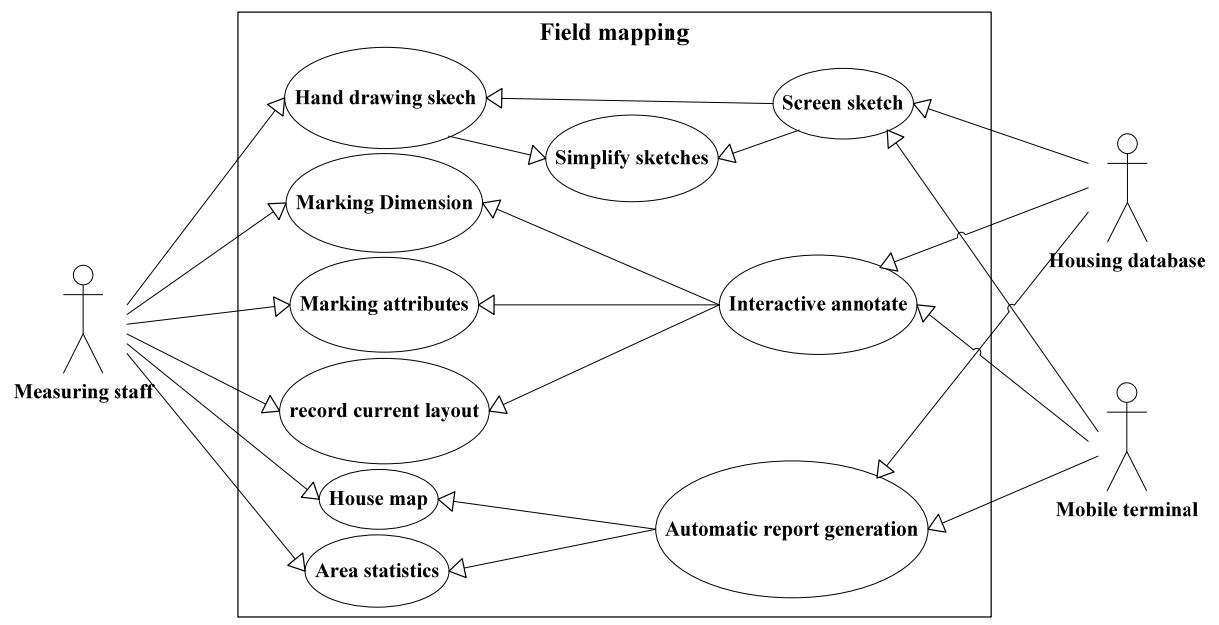

Fig. 2 The Use Case Diagram

\subsection{Software Architecture Design.}

The parametric mapping software is designed to realize the fast mapping in the fieldwork. It provides a field mapping methods for measurement staff to drawing while measuring and interactive designation and processing data in synchronous. With reference to the Real Estate Measurement Specification [4] and fieldwork experience, the system framework is shown in Figure 3.

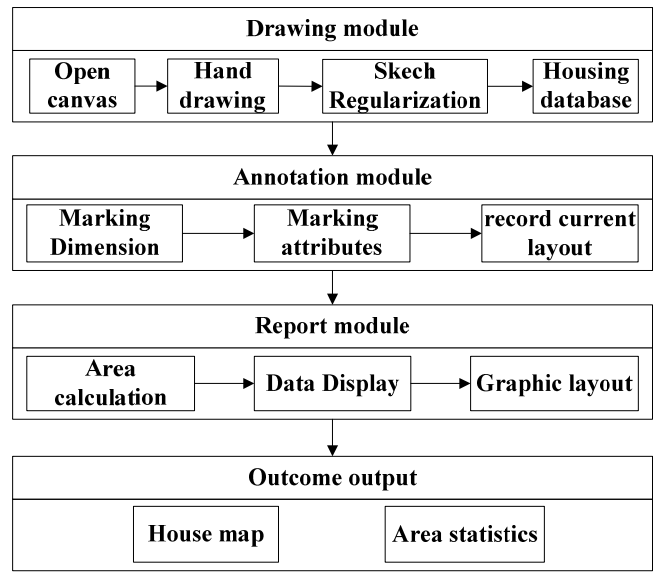

Fig. 3 System Structure

The software has three major modules: drawing module, marking module, and report module. The interactions between the modules include the following three points.

(1) the drawing module gets the house database. The system provides a canvas for the surveyor to sketch drawing. After graphics are organized and size chain is generated automatically, the house graphics and dimension chains are stored.

(2) the marking module modifies and consummates the database. The surveyor interactively modifies the dimension chain, adds the attributes, and stores the current general layout.

(3) the report module processes the data and outputs it as a visual result. According to the house size and attribute information; the area is calculated and stored in the database, then the data and graphics are output in the report.

The three functional modules are related to each other through the database and together constitute the core part of the software. 


\section{Software System Implementation}

\subsection{Support for Hand Sketches on the Screen and Automatic Recognition of Sketch Structures.}

To achieve the above functions, it is necessary to overcome the key algorithms of automatic recognition of sketch drawing structures, automatic construction of dimension chains, and automatic redraw of parameters, and implement the above algorithms on mobile platforms [5].

The real estate house map is a plane vector data with no coordinate system [6]. The drawing function mentioned in this article is implemented in the screen coordinate system. Sketch drawings are the most intuitive and original expression of the size of the measured house and the relative position between the houses. The implementation process of the screen sketch drawing function is shown in Figure 4, the Android system provides a Canvas drawing mechanism [7], and the real-time drawing of the field sketch is a $2 \mathrm{D}$ drawing. When the user is drawing on the screen, the finger pressing action triggers the Android gesture recognition method, the system recognizes the gesture track, collects the slide track coordinate points in real time, and passes the coordinates to the on Draw method of the canvas, and draws the real-time sampling points onto the canvas. The drawing stops when the finger is lifted and a set of points is obtained, that is, vector strokes.

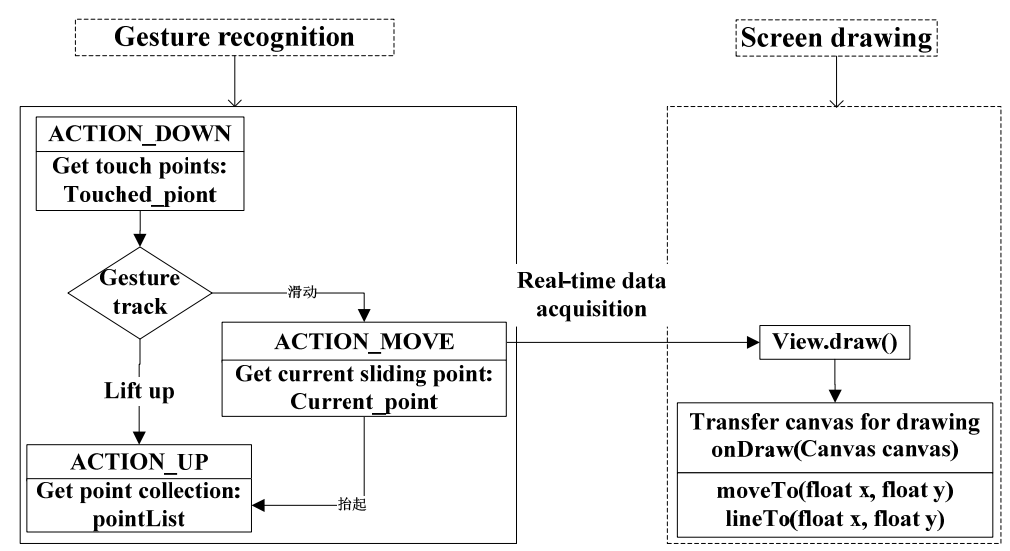

Fig. 4 The Realization Process of Screen Hand Drawing

After obtaining the vector sketch of the house, the sketch is simplified by the system to fit the actual house drawing. As shown in Figure 5, the core algorithm is the sketch auto-reduction algorithm. The algorithm implementation process is described as follows:

(1) Vector data compression. Douglas-Puker algorithm is used to compress a large number of hand-drawn stroke data points, simplifying into line segments. Under the resolution of $2560 * 1600$ pixels for Android devices, it is recommended to set a threshold value of 40 pixels (approximately $3.4 \mathrm{~mm}$ ) to remove small bends and retain large bends, which conforms to the drawing intention.

(2) Graphic closure. According to the different conditions of the intersection, an intersection algorithm is programmed to intersect the vector-compressed segments. Only the intersections are reserved to remove the intersections of the segments. The gaps, whose thresholds are smaller than the threshold automatically close, for those which are larger than the threshold, require the user to redraw.

(3) Using two clustering algorithms (K-Means, $K=2$ ) [8] for each closed graph to find the two main directions of the graph. This paper clusters the main distribution trends of line segments: (1) Get the directional angle of all line segments and horizontal direction. (2) Select line segments with $1 / 3$ and $2 / 3$ of the maximum and minimum values of the direction angle as the initial centroids of the two clusters. (3) Traversing all line segments to determine the angle difference between the current line segment and the two centroids respectively. The line segments are categorized into clusters of smaller differences, so that line segments with similar direction angles are clustered into the same cluster and two line segment clusters. (4) For each of the two clusters, separately calculate a mean line segment of the direction angle to replace the initial centroid as the representative direction of the cluster and becomes two new centroids. (5) Repeat (3)(4), Until the current difference between the 
centroid and the upper centroid is less than a constant close to 0 , stopping the clustering, and the current centroid is the 2 main directions.

(4) Rotate the two main directions to be perpendicular to each other. Rotate the edges with smaller angles from the main direction in the closed graph to the main direction. For those with larger angles, retain their irregular characteristics and generate closed orthogonal polygons.

(5) Rotate the graphic to make the main direction of the graphic parallel to the screen coordinate system, and to obtain the actual house graphic.

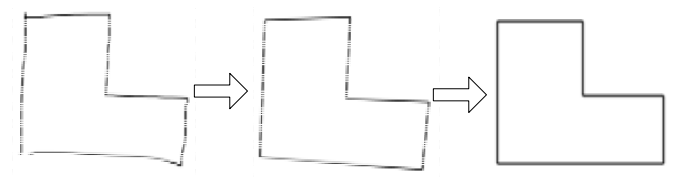

Fig. 5 Schematic Diagram of Automatic Simplification Algorithm

After the sketch is reduced, the topological structure is automatically identified. When other transform operations are performed on the closed graph, the topology remains unchanged. The core algorithm is the contour tracking algorithm. The contour tracking algorithm in this paper is based on vector graphics. The algorithm process is described as follows. As shown in Figure 6, AB is the starting line segment: (1) Set the start point of the starting segment as the contour tracking start point. (2) Follow the clockwise direction to get the vector 1 where the starting point points to the end point. (e.g. $\overrightarrow{A B}$ ) (3) Mark the end point as the current tracking point, and the subsequent point target set is obtained, and separately obtain the vector 2 of the target point pointing to the current tracking point. (e.g. $\overrightarrow{E B}, \overrightarrow{G B}, \overrightarrow{C B}$ ) (4) Find the maximum clockwise angle between vector 1 and vector 2 , the target point of the current vector 2 is the next tracking point. (If now the clockwise angle between $\overrightarrow{A B}$ and $\overrightarrow{C B}$ is the largest and $\mathrm{C}$ is the next tracking point.) (5) Repeat step (3) and (4), if the target point coincides with the initial point, the algorithm stops and obtains the contour. (E.g. profile ABCD.)

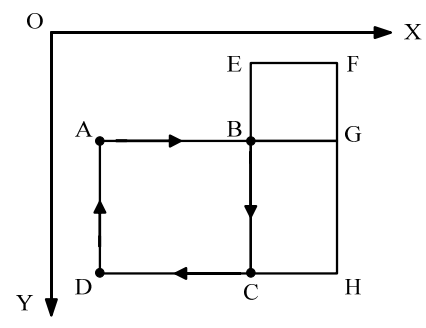

Fig. 6 Tracking Algorithm Schematic

\subsection{House Database Storage and Dimension Chain Generation.}

Android supports the creation and use of SQLite database, which is lightweight and suitable for mobile device use [9]. The database model for storing house graphics consists of three entity tables: point, line, and house. A point is recorded on every row of the point table. Its attributes include point number, abscissa and ordinate. The coordinates of points are coordinates of the drawing coordinate system; A line segment is recorded in each row of the line table, and its attribute content includes start point number, end point number, annotation location, and labeling state; The housing table records one surface each line. Its attribute content includes the house number, outline number, floor, area calculation coefficient, area, and house structure. The area calculation coefficient is determined by the properties of the house and is divided into the calculation of all building areas (assignment 1) Calculation of half of the area (assignment 0.5) and the area of non-calculation (assignment 0). The function of the house database includes creating tables of points, lines, and surfaces, adding, querying, and updating the house database. This data model can be used to draw information from the line segment table on the canvas by calling the line.db file of the SQLite database to satisfy the needs of house graphics storage. 
After completion of hand drawing on the screen, the graph will automatically set up house dimension chain with horizontal and vertical compound chains used for expression. Each node of compound chain stores collinear shape points: Each node of horizontal compound chain table stores collinear points in the horizontal direction. For example, point A and point B are collinear in the first node of Figure 7(a); each node of vertical compound chain table stores collinear points in the vertical direction. For example, point $A$ and point $F$ are collinear in the first node of Figure 7(b).

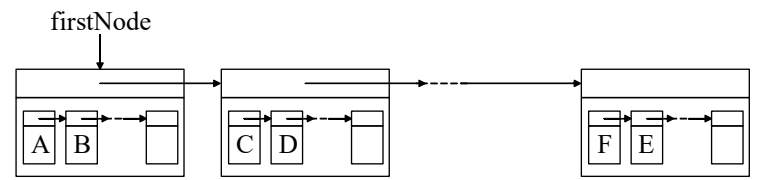

(a) Horizontal Chain Expression Method

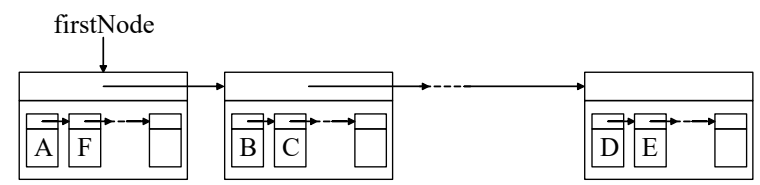

(b) Vertical Chain Expression Method

Fig. 7 Description of Dimension Chain LinkedList

Establish the dimension chain data file in the database, execute SQL sentences and insert them into the database one by one as well as record dimension and starting point number of each dimension chain.

\subsection{Parametric Input and Update.}

According to the interaction event on Android devices [10], the interactive annotation function is designed. Respond to self-defined click event, long press event, control click event as well as trigger tagging function dialog box by realizing OnClickListener interface [11]. As shown in the Figure 8: (1) Trigger dimension input dialog box to record dimension by house contour line pickup algorithm with drawText() method in the Canvas used to draw characters in the middle on one side of the line segment for completing annotation: (2) Trigger self-defined attribute dialog box by planar graph pickup algorithm and select input attribute information in Spinner control; (3) Enable the camera function dialog box to record the current general layout of house with photo recorded into local folders. After completing all annotations, field operation data acquisition will end.

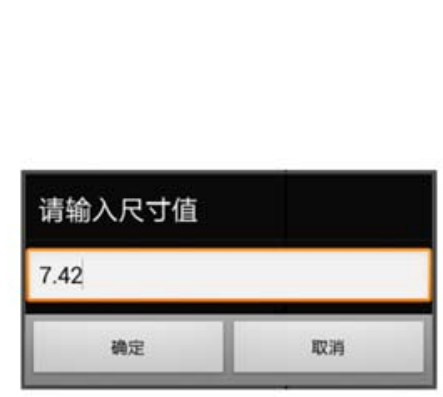

(a) Dimension Marking

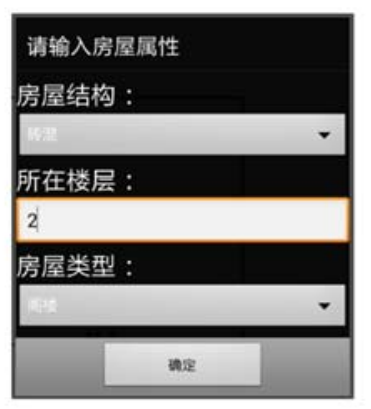

(b) Attributes Marking

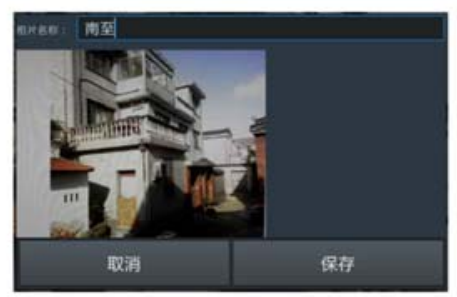

(c) Four Orientations

Fig. 8 Mark Pattern Dialog

Automatically update house dimension chain after inputting dimension driven drawing dimension data and realizing dimension driven drawing [12]. Algorithm flow is as follows (taking horizontal dimension chain as an example): (1) As shown in Figure 9, input horizontal size L on AB side as well as seeking horizontal dimension chain with starting point A. (2) Find the node where A is, modify the coordinate of end point B and update it to B'. (3) Seek vertical dimension chain with B, find C, modify the coordinate of point $C$ and update it to $C^{\prime}$. (4) Seek horizontal dimension chain with $C$, find $D$ and update it to D'. (5) Repeat (3) (4) Until point F and starting point A are on the same node in the vertical dimension chain, point F remains unchanged and seeking ends. Drawing of graph anew is completed. 


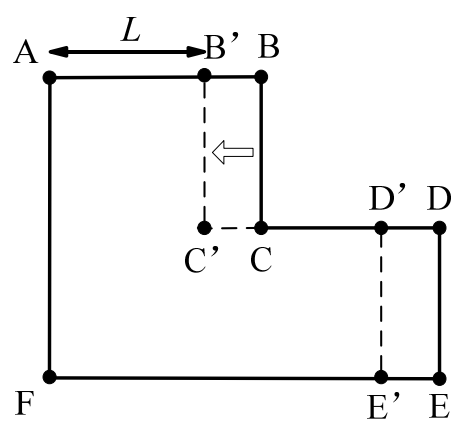

Fig. 9 Schematic Diagram of Dimension-Driven Drawing

A few of irregular house plans arise in the house property survey. In case of hypotenuse size marking, parameter-driven drawing as per input hypotenuse dimension shall be conducted. For one hypotenuse, confirm small endpoint of y-coordinate as fixed point. There are two kinds of hypotenuse dimension driving-modification of horizontal dimension chain and modification of vertical dimension chain.
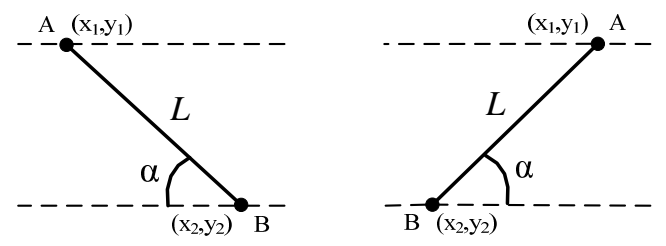

(a) Modify the Horizontal Dimension Chain
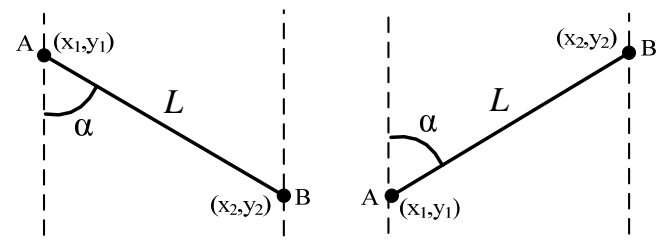

(b) Modify the Vertical Dimension Chain

Fig. 10 Beveling Dimension-Driven Drawing

As shown in Figure 10 (a), small point of y-coordinate is confirmed as starting point, big point of $\mathrm{y}$-coordinate is confirmed as end point. It's necessary to define $\Delta y=y_{2}-y_{1}, \alpha$ as acute angle, input hypotenuse dimension parameter $\mathrm{L}$ as well as end point $\mathrm{x}$-coordinate after driving:

$$
x_{2}^{\prime}=x_{1}+K L \cos \left(\arcsin \frac{\Delta y}{L}\right)
$$

When $x_{2}-x_{1}>0, K=1$. When $x_{2}-x_{1}<0, K=-1$.

As shown in Figure 10(b), small point of $x$-coordinate is confirmed as starting point, big point of $\mathrm{X}$-coordinate is confirmed as end point. It's necessary to define $\Delta x=x_{2}-x_{1}, \alpha$ as acute angle, input hypotenuse dimension parameter $\mathrm{L}$ as well as end point y-coordinate after driving:

$$
y_{2}^{\prime}=y_{1}+K L \cos \left(\arcsin \frac{\Delta x}{L}\right)
$$

When $y_{2}-y_{1}>0, \mathrm{~K}=1$; When $y_{2}-y_{1}<0, \mathrm{~K}=-1$.

\subsection{Real Estate House Graph Generation.}

Report module uses Android Intent to realize report page skipping. The report interface is designed by Android customization layout [13]. In accordance with master drawing design report style set forth in the Specification [14], reports in the paper are divided into the real estate household diagram and area statistics table. The design thought is as follows:

(1) Creation of the real estate household diagram report layout table1.xml.

The real estate household diagram is realized under the user-defined nested $<$ TableLayout $>$ table layout. The report is designed in the form of table and figure. The data is shown in the corresponding position above the table. Finally, disposed housing graph is shown in the subject position below the table. In the data display area, $<$ TableRow $></$ TableRow $>$ is used to realize each row based on sideto-side setup in the table as well as $<$ textview $/>$ and $<$ EditText $>$ in each row are used to display item and input box in the table; in the graphic display area, $<$ ImageView $>$ is used to place house graph.

(2) Creating area statistical table layout table2.xml. 
Area statistical table is realized under the linear layout of $<$ LinearLayout $/>$. ListView in View component is used to display four column names and corresponding value, and each column of listview is realized by one $<$ textview $/>$ as well as long string of the table is realized by $<$ view $/>$ control.

(3) TableAdapter adapter is used to bind data.

(4) Data display in the table is realized in TableActivity.java. Query and invoke data in the database by SQL paging query method, acquire area attribute of area pattern, return one collection and conduct summation of total area. For TextView assignment, the data is displayed to ListView, total area is displayed to household division drawing area bar and the area of each house is displayed to the table as per building number, structure, etc.

(5) Graph display Pursuant to location information and drawing information, the house graph is drawn to Canvas of View by draw method.

Automatic simplification of sketch and parametric dimension driven drawing support realization of free modification of graph. Only feature points for expressing housing graphics are reserved after graphic simplification which shall be numbered. At the moment of modifying the graph each time, it's necessary to confirm the point number to be modified, search point database as per point number as well as modify corresponding point coordinate. As a result, length attribute of one segment is modified, corresponding segment gets new length attribute, dimension parameters in the graph changes, endpoint coordinate changes accordingly, the database is automatically updated, data in the table is updated accordingly. So chart linkage is realized.

Final house graph and data are output into maps based on DWG format as well as the house map and area statistical table based on PDF format. These can be used as the results of real estate fieldwork. In the end, the results are uploaded to office work promptly for writing house report.

\subsection{Software Interface Introduction.}

For the purpose of realizing related functions of the software, the paper adopts nested Relative Layout to design interactive UI interface [15]. Main interface of household division drawing field work mapping software is shown in Figure 11, including menu bar, tool bar and drawing area. Tool bar covers three modules: drawing module, marking module and report module.

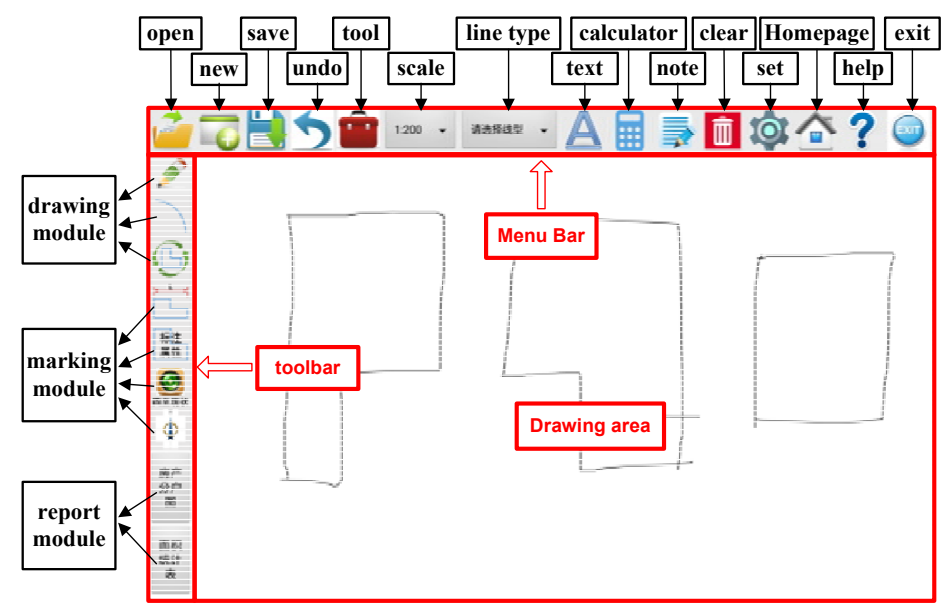

Fig. 11 Main Interface

Software operation step is as follows:

(1) After entering the survey area, the surveyor observes the house, carry out direct freehand sketching of house on the screen, enable tool bar and click sketch optimization button to get regular house sketch. Initial annotation is automatically generated at the same time which is marked in the middle of one side of house contour line.

(2) After completing drawing house graph, pickup any building border line to input actual dimension of such line and modify size marking with dimension driven drawing. After completing 
measuring each house, press the house for a long time to input house attribute information and display it for corresponding house at the same time.

(3) After marking all dimension and attribute information, open the report module and then get the house map and area statistical table.

\section{Experiments and Analysis}

To test the function and performance of the software described in this paper, field experiments and comparative experiments were designed.

\subsection{Function Test.}

the houses in one measurement area of Suzhou are selected for house fieldwork experiment to test software functions. Most of houses in the area are new rural housing characterized by independent construction for each household and building structure is relatively regular. For area calculation, only property area of each household is required to be calculated without area apportions calculation involved. Taking one household as an example, this household has true production data which can be used for inspection of experimental result in the paper.

House sketch is shown in Figure 11. House information are marked one by one on the regular graph part of the house map (Figure 12). The graphic part records current situation of spatial distribution, dimension and attribute information which can be used to calculate area and prepare report after affirming to be qualified by process check and final inspection.

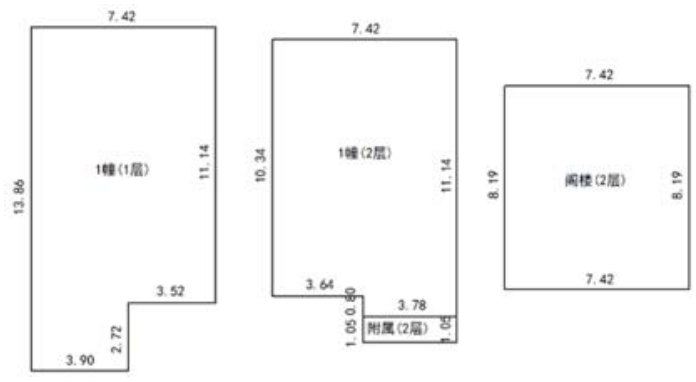

Fig. 12 Hand-Painted House Graphics of the Example

Figure 13 is the house map and area statistical table under the report module. Some important conclusive data are displayed to the notable location. Land number, building number and door number, etc. are filled in the table. The main body displays house graph as well as the area of each house can be provided to the owner for check.

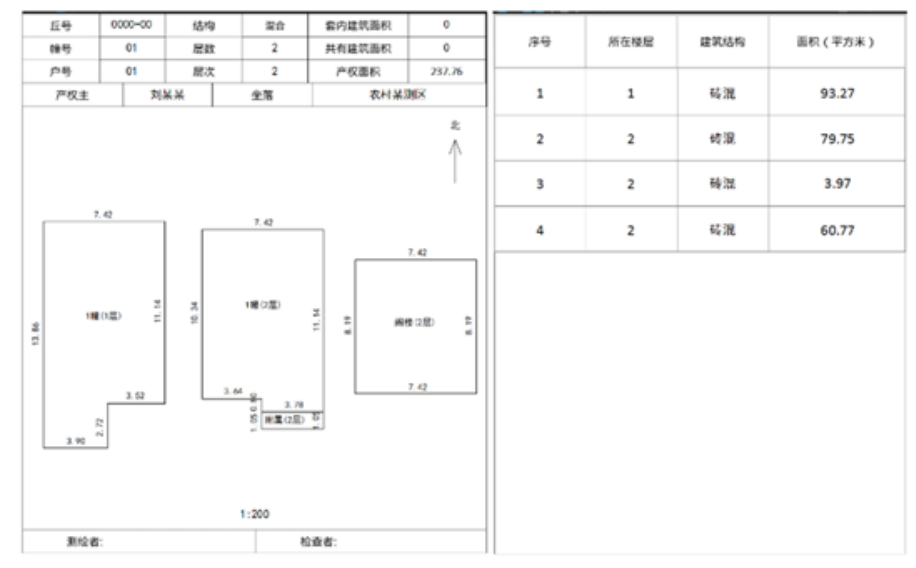

Fig. 13 House Map and Area Statistics

Experiments show that the software output results are correct compared with the actual production data. The function of the software in this paper can achieve the expected results. After the output report is accepted by the inspection agency, the surveying technical report can be written. 


\subsection{Performance Test.}

In the land requisition and demolishing work in one village of Nanjing, houses are selected for contrast experiment to test software performance. Each household in the area is independently built and is in possession of many houses with large workload of fieldwork.

Traditional method and software method in the paper are used to measure four house properties, including 24 houses. After comparing the time needed by two methods, software performance is tested. As shown in Figure 14, traditional method takes about 3 hours from drawing fieldwork sketch, house data acquisition and office work to calculation of house area; the method in the paper takes about 1.5 hours from completion of hand drawing, data acquisition and parametric drawing to report generation. It decreases the workload.

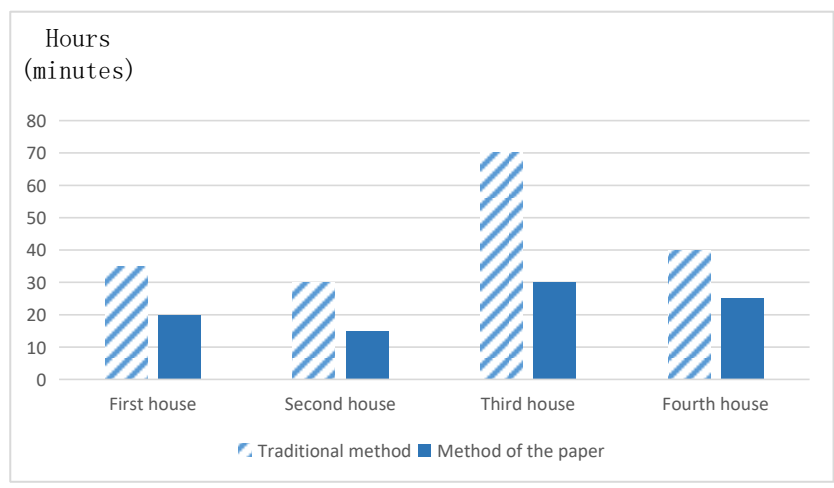

Fig. 14 Measuring Time Contrast Figure of Two Ways

According to the experiment, the software is conveniently operated. Sketch drawing of fieldwork can be directly applied to generate the house map with measurement and drawing at the same time so as to timely find and correct mistakes. Integrative calculation method avoids error of artificial calculation, reduces working intensity and improves calculation accuracy. Final result is displayed in the report module in the form of table with figure. The real estate measurement can be quickly completed.

\section{Summary}

In view of the situation of rural housing frequent changes and time-consuming data updating, the development of the house mapping software is put forward in this paper. Firstly, a real-time field mapping technical process based on automatic drafting of sketches is designed. And key algorithms such as automatic sketch drawing recognition, the automatic construction of the dimension chain and the parametric self-construction are studied and realized. The Android platform software with the above functions is developed. Finally, experiment is designed to verify the function and performance of the system. Results show that the software is intuitive and convenient to use. The efficiency of the real estate measurement in China is greatly improved.

\section{References}

[1]. Yang Bogang, Zhang Baogang. Current Situation and Analysis of Real Estate Surveying and Mapping in China. Bulletin of Surveying and Mapping. (2016) No. 12, p. 1-6.

[2]. Zheng Jinshui. On the Property Management of Surveying and Mapping Under the New Situation. Journal of Geomatics. Vol. 40(2015) No.04, p. 87-89.

[3]. Xin Yizhong, Li Yan, Yuan Weiqiang, et al. Comparison of target selection techniques in mobile handheld environment. Journal of Image and Graphics. Vol. 20(2015) No. 02, p. 264-270.

[4]. The state bureau of surveying and mapping[S]. GB/T 17986.1-2000. Specifications for estate surveying-Unit 1: General rules for estate surveying. 2000. 
[5]. Zhang Honghua. The retrieval method and system of the topographic map sheet information on android platform. Science of Surveying and Mapping. Vol. 11(2017) No.42, p. 184-187.

[6]. Kong Qingfu, DU Ao. The 3D Management Research Based on the Data of Real Estate Registration Data Integration. Bulletin of Surveying and Mapping. (2017)No.S2, p. 146-148.

[7]. Jackson W. Interactive Drawing: Using Paint and Canvas Classes Interactively. Pro Android Graphics. (2013), p. 479-513.

[8]. Kishor D R, Venkateswarlu N B. Hybridization of Expectation-Maximization and K-Means Algorithms for Better Clustering Performance. Cybernetics and Information Technologies. Vol.16 (2016) No.02, p. 16-34.

[9]. Wang G, Zhao M Y. Study and Optimization of Database on Android Platform. Applied Mechanics and Materials. (2013), p. 1754-1758.

[10]. Zhang Guoyin, Qu Jiaxing, Fu Xiaojing, et al. Android Malicious Behavior Detection Method Based on Composite-event Trigged Behaviors. Computer Science. Vol. 05(2016) No.43, p. 96-99.

[11]. Griol D, Molina J M. A Framework to Develop Adaptive Multimodal Dialog Systems for Android-Based Mobile Devices. Hybrid Artificial Intelligence Systems. (2014), p. 25-36.

[12]. Yin S J, Sun M Q, Li X, et al. Research and Application of Parametric Design in Traditional Plug-Flow Aeration Tank with Drawing Methods. Advanced Materials Research. Vol. 10(2014) No.14, p. 86-89.

[13]. Ludin A. ListView and DataModel. Learn BlackBerry 10 App Development. (2014), p. 171218.

[14]. The state bureau of surveying and mapping[S]. GB/T 17986.2-2000. Specifications for estate surveying-Unit 2: Specifications for symbols of estated map. 2000.

[15]. Neil T. Mobile Design Pattern Gallery: UI Patterns for Mobile Applications. America: O'Reilly Media Inc. (2014). 